\title{
QUASICONFORMALLY HOMOGENEOUS PLANAR DOMAINS
}

\author{
PETRA BONFERT-TAYLOR AND EDWARD C. TAYLOR
}

\begin{abstract}
In this paper we explore the ambient quasiconformal homogeneity of planar domains and their boundaries. We show that the quasiconformal homogeneity of a domain $D$ and its boundary $E$ implies that the pair $(D, E)$ is in fact quasiconformally bi-homogeneous. We also give a geometric and topological characterization of the quasiconformal homogeneity of $D$ or $E$ under the assumption that $E$ is a Cantor set captured by a quasicircle. A collection of examples is provided to demonstrate that certain assumptions are the weakest possible.
\end{abstract}

\section{INTRODUCTION}

Recall that a hyperbolic manifold is uniformly quasiconformally homogeneous if there exists a constant $K \geq 1$ so that any two points on the manifold can be paired by a $K$-quasiconformal automorphism. Owing to quasiconformal rigidity phenomena there exists a complete topological and geometric understanding of uniformly quasiconformally homogeneous hyperbolic manifolds [2] in dimensions three and above.

The situation in dimension two is richer (e.g. see [1], 4], and [3]), and our purpose in this paper is to explore various notions of quasiconformal homogeneity in the plane. In doing so we are returning to the origins of the theory of quasiconformal homogeneity as initiated by Gehring and Palka in [6], and continued by MacManus, Näkki and Palka in 12 as well as [13]. Indeed, our work in this paper builds upon their work.

We now focus our discussion on sets in the Riemann sphere $\hat{\mathbb{C}}$. The basic definition is: A set $A \subset \hat{\mathbb{C}}$ is $K$-quasiconformally homogeneous if for every $x, y \in A$ there exists a $K$-quasiconformal homeomorphism $f: \hat{\mathbb{C}} \rightarrow \hat{\mathbb{C}}$ such that $f(A)=A$ and $f(x)=y$. If such a $K$ exists for $A$, we also call $A$ uniformly quasiconformally homogeneous.

In this paper $A$ will always be either a domain $D$ (i.e. an open and connected set) with at least three boundary points or $A$ will be the complement $E$ of such a domain. We equip the domain $D$ with the hyperbolic metric.

If $D=\Omega(\Gamma)$ is the regular set associated to a Kleinian Schottky group $\Gamma$, then it is uniformly quasiconformally homogeneous via an application of Theorem 1.3 in [2]. Observe as well that it is a result of MacManus, Näkki and Palka 12] that the limit set $E=\Lambda(\Gamma)$ of such a group $\Gamma$ is an example of a compact set that is uniformly quasiconformally homogeneous. The pair $(\Omega(\Gamma), \Lambda(\Gamma))$ exhibits

Received by the editors June 19, 2008.

2000 Mathematics Subject Classification. Primary 30C62; Secondary 30F45.

Both authors were supported in part by NSF grant DMS 0706754.

(C)2008 American Mathematical Society Reverts to public domain 28 years from publication 
([13]) a seemingly stronger form of joint quasiconformal homogeneity (called quasiconformal bi-homogeneity, see Section 2), where one can simultaneously, with one quasiconformal automorphism, pair both two points in $\Omega(\Gamma)$ and two points in $\Lambda(\Gamma)$.

We will investigate in general the relation between quasiconformal homogeneity of a domain $D$ and of its complement $E$. The "scorecard" below summarizes the possible outcomes. Our first result gives the entry in the upper left hand corner of the scorecard:

Theorem 1.1. Suppose that $E$ is a compact set in $\hat{\mathbb{C}}$ with more than two points so that its complement $D$ is connected. Then the following are equivalent:

(1) Both $D$ and $E$ are individually uniformly quasiconformally homogeneous, and

(2) the pair $(D, E)$ is quasiconformally bi-homogeneous.

Clearly (2) implies (1), and so the content of the theorem is that quasiconformal bi-homogeneity only appears to be stronger than the separate homogeneities of $D$ and $E$. Using the refined analysis of quasiconformally bi-homogeneous pairs in [13] we can give a full geometric characterization of any pair $(D, E)$ that lives in the upper left hand corner of the scorecard.

Not every pair $(D, E)$ is quasiconformally bi-homogeneous, even if one of $D, E$ is quasiconformally homogeneous. The following scorecard organizes examples that illustrate the possible outcomes. The abbreviation "uqch" stands for "uniformly quasiconformally homogeneous." Recall that $D$ is a domain in $\hat{\mathbb{C}}$ with complement $E=\hat{\mathbb{C}} \backslash D$.

TABLE 1. Planar QC Homogeneity Scorecard

\begin{tabular}{|l|l|l|}
\hline & $D$ is uqch & $D$ is not uqch \\
\hline E is uqch & $\begin{array}{l}\text { completely classified, see } \\
\text { Theorem 1.1 and Corol- } \\
\text { lary 2.1 }\end{array}$ & Example 3.3 \\
\hline E is not uqch & Example 3.1 & Example 3.5 \\
\hline
\end{tabular}

The table records that quasiconformal homogeneity of $D$ can be decoupled from quasiconformal homogeneity of $E$. However, if $E$ is captured by a quasicircle, then these notions of quasiconformal homogeneity can no longer be decoupled.

Theorem 1.2. Let $D \subset \hat{\mathbb{C}}$ be a domain such that $E=\hat{\mathbb{C}} \backslash D$ is contained in a quasicircle. Then the following are equivalent:

(1) $D$ is uniformly quasiconformally homogeneous.

(2) E is uniformly perfect and uniformly quasiconformally homogeneous.

(3) $(D, E)$ is quasiconformally bi-homogeneous.

(4) The injectivity radius function on $D$ is bounded above and away from zero. 
We provide examples (see Remark 3.4) that illustrate that uniform perfectness in (2) is indeed a necessary assumption and is not implied by the uniform quasiconformal homogeneity of $E$. On the other hand, we prove in [5] that in the setting of Kleinian groups, uniform quasiconformal homogeneity of a limit set always implies uniform perfectness of the limit set, independent of whether or not the limit set can be captured by a quasicircle. In fact, in 5. we completely classify those limit sets of Kleinian groups that are uniformly quasiconformally homogeneous.

We also note that the implication $(4) \Rightarrow(1)$ in the above theorem is special to this setting. It is easy to produce examples of surfaces with bounded geometry that are not uniformly quasiconformally homogeneous; see Example 4.2 .

A final word concerning our definition of uniform quasiconformal homogeneity: By using quasiconformal mappings of the entire sphere we are avoiding delicate issues (see for example [7, 8, 9, 10]) concerning quasiconformal removability. In the paper [3] we call such sets ambiently quasiconformally homogeneous.

\section{EqUiVAlence}

We recall a definition established in 13 . Let $D$ be a domain in $\hat{\mathbb{C}}$ and let $E$ be its complement. The pair $(D, E)$ is quasiconformally bi-homogeneous if there exists a $K \geq 1$ so that for all pairs $(z, w) \in D$ and $(s, t) \in E$ there exists a $K$-quasiconformal homeomorphism $f$ of $\hat{\mathbb{C}}$ that keeps $D$ invariant and so that $w=f(z)$ and $t=f(s)$. For ease of terminology we are not inserting the word "uniformly" in the definition of quasiconformally bi-homogeneous, though it is clearly implied. Also, it is obvious that if the pair $(D, E)$ is quasiconformally bi-homogeneous, then individually both $D$ and $E$ are themselves uniformly quasiconformally homogeneous.

The condition of quasiconformal bi-homogeneity for the pair $(D, E)$ is only apparently stronger than the quasiconformal homogeneity of both $D$ and $E$ individually. For convenience, we restate Theorem 1.1

Theorem. Suppose that $E$ is a compact set in $\hat{\mathbb{C}}$ with more than two points so that its complement $D$ is connected. Then the following are equivalent:

(1) Both $D$ and $E$ are individually uniformly quasiconformally homogeneous, and

(2) the pair $(D, E)$ is quasiconformally bi-homogeneous.

Before we prove this theorem, we record the geometric information we can conclude as a consequence from the above theorem, following [13.

Corollary 2.1. Let $D, E$ be as above, and suppose that both $D$ and $E$ are uniformly quasiconformally homogeneous. Then this is equivalent to each of the the following two statements:

(1) $E$ is quasiconformally equivalent to the middle $1 / 3$ Cantor set, and

(2) $E$ is uniformly perfect and $D$ is a uniform domain.

Recall that a domain $D \subset \mathbb{C}$ is uniform if there exists a constant $c>1$ so that for each pair of distinct points $a, b \in D$ there exists a path $\beta \in D$ joining $a$ and $b$ such that the Euclidean diameter of $\beta$ is less than or equal to $c|a-b|$ (here $|a-b|$ is the Euclidean distance of $a$ to $b$ ), and

$$
\bigcup_{z \in \beta \backslash\{a, b\}} B\left(z, \frac{\min \{|z-a|,|z-b|\}}{c}\right) \subset D .
$$


Furthermore, a domain $D$ in $\hat{\mathbb{C}}$ is uniform if $D \cap \mathbb{C}$ is uniform. Recall also that a compact set $E$ in $\hat{\mathbb{C}}$ is uniformly perfect, if the moduli of all separating annuli are uniformly bounded from above.

Proof of Theorem 1.1. Clearly (2) implies (1), and so all we have to show is that (1) implies (2). To see this, we will first show the following.

We claim: Uniform quasiconformal homogeneity of $E$ implies that for a fixed $z_{0} \in D$ there exists a constant $K_{1}$ so that for any two points $s, t \in E$ there exists a $K_{1}$-quasiconformal mapping $h$ of $\hat{\mathbb{C}}$ that keeps $E$ invariant, maps $s$ to $t$, and that fixes $z_{0}$. To prove this claim, we use an argument adapted from [12]: Let $\mathcal{F}$ be the family of all quasiconformal mappings of $\widehat{\mathbb{C}}$ that fix $E$ setwise and that also fix $z_{0}$. Then $\mathcal{F}$ acts transitively on $E$ since for any $s, t \in E$ there exists a quasiconformal $f: \hat{\mathbb{C}} \rightarrow \hat{\mathbb{C}}$ with $f(E)=E$ and $f(s)=t$ by quasiconformal homogeneity of $E$. The map $f$ does not necessarily fix $z_{0}$, but we may postcompose $f$ with a quasiconformal mapping $g: \hat{\mathbb{C}} \rightarrow \hat{\mathbb{C}}$ that is the identity on $E$ and maps $f\left(z_{0}\right)$ to $z_{0}$ (see [6] and [2] for example). Thus $g \circ f \in \mathcal{F}$, and so $\mathcal{F}$ indeed acts transitively on $E$. Now let $\mathcal{F}_{n}=\{f \in \mathcal{F} \mid K(f) \leq n\}$, then $\bigcup_{n=1}^{\infty} \mathcal{F}_{n}=\mathcal{F}$. Fix $x \in E$, and let $E_{n}=\mathcal{F}_{n}(x)$. Then $E=\bigcup_{n=1}^{\infty} E_{n}$, and since each $f \in \mathcal{F}_{n}$ fixes $z_{0}$ as well as the set $E$ and has dilatation at most $n$, the family $\mathcal{F}_{n}$ is compact, and hence $E_{n}$ is closed. The Baire Category Theorem ensures that at least one of the $E_{n}$, say $E_{N}$, has an interior point in the relative topology of $E$. Thus there exists an open set $U \subset \hat{\mathbb{C}}$ such that $U \cap E \subset E_{N}$. Compactness of $E$ now implies that there exist $f_{1}, \ldots, f_{p} \in \mathcal{F}$ such that $\left\{f_{1}(U), \ldots, f_{p}(U)\right\}$ is an open cover of $E$. Let now $s, t \in E$. Then there exist $k, l \in\{1, \ldots, p\}$ and $\tilde{s}, \tilde{t} \in U \cap E \subset E_{N}$ such that $s=f_{k}(\tilde{s})$ and $t=f_{l}(\tilde{t})$. Since also $\tilde{s}, \tilde{t} \in E_{N}$, there are $g_{1}, g_{2} \in \mathcal{F}_{N}$ such that $\tilde{s}=g_{1}(x)$ and $\tilde{t}=g_{2}(x)$. Setting $h=f_{l} \circ g_{2} \circ g_{1}^{-1} \circ f_{k}^{-1}$ we see that $t=h(s)$, where $h \in \mathcal{F}$ and $K(h) \leq K_{1}=N^{2} \cdot \max \left\{K\left(f_{1}\right), \ldots, K\left(f_{p}\right)\right\}$. This proves the claim.

Now, using the uniform quasiconformal homogeneity of $D$ and the fact we just proved we see that there exists a constant $K_{2}$ such that for any $z \in D$ and any $s, t \in E$ there exists a $K_{2}$-quasiconformal mapping $g$ of $\hat{\mathbb{C}}$ that keeps $E$ invariant, maps $z$ to $z_{0}$, and maps $s$ to $t$ (first map $z$ to $z_{0}$ with some $f$ using the uniform quasiconformal homogeneity of $D$, then map $f(s)$ to $t$ while fixing $z_{0}$ as above).

Finally, let $s, t \in E$, and let $z, w \in D$. Then by the above there exist $K_{2}$ quasiconformal mappings $g_{1}, g_{2}$ of $\hat{\mathbb{C}}$ that keep $E$ invariant, and so that $g_{1}$ maps $s$ to $t$ and $z$ to $z_{0}$, and so that $g_{2}$ maps $t$ to $t$ and $w$ to $z_{0}$. Thus the mapping $g_{2}^{-1} \circ g_{1}$ fixes $E$, maps $z$ to $w$, and $s$ to $t$, while its dilatation is at most $K_{2}^{2}$. Hence $(D, E)$ is quasiconformally bi-homogeneous.

\section{The EXAmple ZOO}

The assumption of quasiconformal bi-homogeneity is restrictive. Here, via examples, we fill in a portion of our scorecard that demonstrates that a domain $D$ can be uniformly quasiconformally homogeneous without its complement $E$ being so, and vice versa. Note that in [12] it is shown that if $E$ is a uniformly quasiconformally homogeneous compact proper subset of $\hat{\mathbb{C}}$, then either $E$ has only finitely many points, or $E$ is the union of a finite collection of disjoint non-nested quasicircles, or $E$ is a Cantor set of Hausdorff dimension strictly less than two. Here, a Cantor set is a compact, totally disconnected, and perfect set. In our setting, with $E$ being the complement of a domain $D$, the only way that gives both $D$ and $E$ a chance 
of being uniformly quasiconformally homogeneous is thus for $E$ to be a Cantor set of Hausdorff dimension less than two (if $E$ is a finite set of points, then on $D$ the hyperbolic injectivity radius function is not bounded from below and thus $D$ cannot be quasiconformally homogeneous, whereas if $E$ is the union of quasicircles, then $D$ is not connected).

Example 3.1. There exists a uniformly quasiconformally homogeneous domain $D$ whose complement $E$ is a uniformly perfect Cantor set of dimension strictly less than two that itself is not quasiconformally homogeneous.

Before constructing this example, we recall that, informally, a John domain is a domain $D$ for which there exists a point $a \in D$ such that any point $b \in D$ can be reached from $a$ by a flexible cone with definite angle at $b$. Uniform domains are examples of John domains, but not every John domain is a uniform domain. One can find an extensive discussion of the John condition in [16. In [15, Theorem 3.1], McMullen classifies the regular sets of finitely generated Kleinian groups that are John domains:

Theorem 3.2 (McMullen). Let $D$ be a component of the regular set of a nonelementary, finitely generated Kleinian group $\Gamma$. Then the following are equivalent:

(1) $D$ is a John domain

(2) The stabilizer $\Gamma_{D}$ of $D$ is geometrically finite, and every parabolic in $\Gamma_{D}$ stabilizes a round disk in $D$.

Construction of Example 3.1. Let $\Gamma$ be the Klein combination of two rank-2 parabolics, i.e. $\Gamma=(\mathbb{Z}+\mathbb{Z}) \star(\mathbb{Z}+\mathbb{Z})$. Let $D$ be the regular set of $\Gamma$. Then there exists a compact fundamental domain for the action of $\Gamma$ on $D$ and thus $D$ is uniformly quasiconformally homogeneous [2]. Furthermore, $E=\partial D=\Lambda(\Gamma)$ is a uniformly perfect Cantor set of dimension strictly less than two. However, no parabolic element of $\Gamma$ stabilizes a round disk in $D$, and thus $D$ is not a John domain by Theorem 3.2 This implies that $D$ is not uniform, and hence $E$ cannot be quasiconformally homogeneous, since otherwise Theorem 1.1 would imply that $(D, E)$ is bi-homogeneous and thus by Corollary 2.1, $D$ would have to be uniform.

Now we provide an example of a domain whose boundary is quasiconformally homogeneous, however the domain is not. This fills in the upper right hand corner of the scorecard.

Example 3.3. Let $d \in[0,2)$, then there exists a Cantor set $E$ of dimension $d$, so that $E$ is uniformly quasiconformally homogeneous but its complement $D$ is not.

Construction of Example 3.3. We first note that in 12 the authors construct a Cantor set $E$ of dimension 0 which is quasiconformally homogeneous (see Example 3.5 in their paper). Such a set $E$ cannot be uniformly perfect since every uniformly perfect set has positive dimension, and thus the injectivity radius function on its complement $D$ is not bounded away from zero which implies that $D$ cannot be uniformly quasiconformally homogeneous by 2]. Thus we are left with having to construct examples of positive dimension. The idea is to construct a uniformly quasiconformally homogeneous Cantor set $E$ that is not uniformly perfect, but has positive dimension. The same reasoning as above then shows that its complement $E$ is not uniformly quasiconformally homogeneous. 
We first describe a standard construction of a Cantor set in the plane that is uniformly perfect and uniformly quasiconformally homogeneous and that has a given dimension $d$ : Let $Q_{0}$ be the square $[-1,1] \times[-1,1]$ in $\mathbb{C}$. Fix $s<1 / 2$, and in each quadrant of $Q_{0}$, center a square of side length $s$ times that of $Q_{0}$. These four new squares form generation 1. Divide each of the four new squares into four equal squares and place a square of side length $s$ times that of the parent centered into each of the four squares. Proceed in a similar fashion. Then in the $n$th step you have a family $\mathcal{F}_{n}$ consisting of $4^{n}$ squares of side length $2 s^{n}$. The intersection of all families $\mathcal{F}_{n}$ yields a uniformly perfect Cantor set of dimension $\log 4 / \log (1 / s)$. This Cantor set is uniformly quasiconformally homogeneous by [12.

Now we modify this construction. In particular, we build in extra shrink factors every now and then, spaced further and further apart such that the resulting Cantor set is no longer uniformly perfect, but it still has dimension $\log 4 / \log (1 / s)$ and it still is uniformly quasiconformally homogeneous.

To be precise, we start out as above with the square $Q_{0}$ of side length 2 , and its four children of side length $2 s$ each (these four children form generation 1 ). In the next step, we shrink each square of generation 1 by a factor $s / 2$, and so we obtain generation 2 which consists of $4^{2}$ squares of side length $2 s^{2} / 2$. To obtain generations 3,4 and 5, we simply shrink the parent square by a factor $s$ each time, to obtain $4^{k}$ squares of side length $2 s^{k} / 2$ for $k=3,4,5$. Now to find generation 6 , we again build in an extra shrink factor, this time we shrink by a factor $s / 3$, so that generation 6 consists of $4^{6}$ squares of side length $2 s^{6} /(2 \cdot 3)$. In general, typically in passing from generation $k-1$ to generation $k$ we shrink by a factor $s$, unless $k$ is of the form $n$ !, in which case we shrink by a factor $s / n$. Hence the $k$ th generation consists of $4^{k}$ squares of side length $2 s^{k} / a_{k}$, where $a_{k}=n$ ! if $n ! \leq k<(n+1)$ !.

Again, let $\mathcal{F}_{n}$ be the union of all $n$th generation squares, and let $C$ be the Cantor set obtained by intersecting all $\mathcal{F}_{n}$.

The $n$th generation squares form an efficient cover of $C$, and so the $\alpha$-dimensional Hausdorff measure of $C$ is

$$
\begin{aligned}
\mathcal{H}_{\alpha}(C) & =\liminf _{k \rightarrow \infty} 4^{k}\left(\sqrt{2} \cdot 2 \cdot \frac{s^{k}}{a_{k}}\right)^{\alpha} \\
& =\liminf _{k \rightarrow \infty} \exp \left(k \log 4+\frac{3 \alpha}{2} \log 2+\alpha k \log s-\alpha \log a_{k}\right) \\
& =\liminf _{k \rightarrow \infty} \exp \left(k \cdot\left(\log 4+\frac{3 \alpha}{2 k} \log 2+\alpha \log s-\alpha \frac{\log a_{k}}{k}\right)\right) \\
& =\liminf _{k \rightarrow \infty} \exp \left(k \cdot\left(\log 4+\alpha \log s+b_{k}\right)\right),
\end{aligned}
$$

where $b_{k} \rightarrow 0$ as $k \rightarrow \infty$, since $a_{k} \leq k$ for all $k$. We observe that if $\log 4+\alpha \log s<0$, then $\mathcal{H}_{\alpha}(C)=0$, whereas if $\log 4+\alpha \log s>0$, then $\mathcal{H}_{\alpha}(C)=\infty$. This implies that

$$
\operatorname{dim} C=\inf \left\{\alpha>0 \mid \mathcal{H}_{\alpha}(C)=0\right\}=\frac{\log 4}{\log (1 / s)} .
$$

The proof in Example 3.5 in [12] still works and shows that our new Cantor set $C$ is uniformly quasiconformally homogeneous. But each square in the $n$ !th generation is contained in the inside component of an annulus of inner radius $\sqrt{2} s^{n !} /(n !)$ and outer radius $s^{n !-1} /(n-1)$ ! that fits into the complement of $C$. The modulus of this annulus is $\log \frac{n}{\sqrt{2} s}$ and tends to $\infty$ as $n \rightarrow \infty$. This implies that $C$ is not uniformly perfect. Thus $C$ is a uniformly quasiconformally homogeneous Cantor 
set of positive dimension which is not uniformly perfect, and so its complement $D$ cannot be uniformly quasiconformally homogeneous.

We record another consequence of the previous example here:

Remark 3.4. There are uniformly quasiconformally homogeneous Cantor sets that are not uniformly perfect. Such sets exist of any dimension $d \in[0,2)$.

We note however that the situation is drastically different when the Cantor set in question is the limit set of a Kleinian group: If the limit set of a Kleinian group is uniformly quasiconformally homogeneous, it must be uniformly perfect; see [5].

For completeness, we provide the following example.

Example 3.5. There exists a domain $D$ with Cantor set complement $E$ so that neither $D$ nor $E$ is uniformly quasiconformally homogeneous.

Construction of Example 3.5. Let $\gamma$ be a hyperbolic element of $P S L(2, \mathbb{R})$ and let $\beta$ be a parabolic element so that the fixed point sets of $\gamma$ and $\beta$ are disjoint. By taking a sufficiently high power of $\gamma$ we can ensure that the group $\Gamma=\langle\gamma\rangle \star\langle\beta\rangle$ is a free discrete non-elementary Fuchsian group of the second kind. We let $E$ be the limit set $\Lambda(\Gamma)$ (necessarily a Cantor set), and we let $D=\hat{\mathbb{C}} \backslash \Lambda(\Gamma)$; that is, $D=\Omega(\Gamma)$.

We first observe that $D$ is not uniformly quasiconformally homogeneous. By the Uniformization Theorem, $D$ is a hyperbolic surface; since $\Gamma$ is geometrically finite (every finitely generated Fuchsian group is geometrically finite) the parabolic fixed points in $E$ are doubly cusped. In particular, there exist infinite area hyperbolic balls in the regular set, which implies that $D$ does not have bounded geometry. By Theorem 1.1 in [2], we observe that $D$ cannot be uniformly quasiconformally homogeneous.

To show that the limit set $E$ is not quasiconformally homogeneous, we show that $E$ is uniformly perfect. The quotient $D / \Gamma$ is topologically the connect sum of a torus with $\mathbb{S}^{1} \times \mathbb{R}$ (see [14, Theorem 7.C.2]) so that if one removes a neighborhood of the cusp, the injectivity radius function is bounded from below. This in turn implies that, off of the horoball neighborhoods of parabolic fixed points, the domain has a lower bound on the injectivity radius. Thus, the whole of $D$ has an injectivity radius bounded from below, and hence $E$ is uniformly perfect. If $E$ were quasiconformally homogeneous, then this would imply by Theorem 1.2 (using that $E$ is contained in $\mathbb{R})$ that $D$ is quasiconformally homogeneous as well, which is a contradiction.

\section{Sets Captured by Quasicircles}

As before, let $D$ be a domain in $\hat{\mathbb{C}}$ and let $E$ be its complement. As we have seen, the homogeneities of $E$ and $D$ can be decoupled. If one adds the additional assumption that $E$ is a subset of a quasicircle, then the situation is quite different. Recall that a quasicircle is the image of the unit circle under a quasiconformal mapping of $\hat{\mathbb{C}}$.

For the convenience of the reader, we restate Theorem 1.2

Theorem. Let $D \subset \hat{\mathbb{C}}$ be a domain such that $E=\hat{\mathbb{C}} \backslash D$ is contained in a quasicircle. Then the following are equivalent:

(1) $D$ is uniformly quasiconformally homogeneous.

(2) $E$ is uniformly perfect and uniformly quasiconformally homogeneous. 
(3) $(D, E)$ is quasiconformally bi-homogeneous.

(4) The injectivity radius function on $D$ is bounded above and away from zero.

Before proving this theorem, we point out a consequence of uniform quasiconformal homogeneity of compact proper subsets of $\mathbb{R}$. We recall that a set $E \subset \mathbb{R}$ is porous in $\mathbb{R}$ if there exists a constant $\lambda>1$ such that every interval $I$ centered on $E$ contains an interval of length $|I| / \lambda$ that lies in $\mathbb{R} \backslash E$.

MacManus, Näkki and Palka [12, Theorem 3.7] prove that a uniformly quasiconformally homogeneous compact set $E \subset \mathbb{R}$ is locally porous in $\mathbb{R}$. We strengthen this result with a refinement of their proof to show that such an $E$ is in fact porous in $\mathbb{R}$.

We first remark that a finite set of points in $\mathbb{R}$ is uniformly quasiconformally homogeneous and porous. Thus we can assume that $E$ is a Cantor set in $\mathbb{R}$ by [12. Theorem 3.3]. As in the proof of Theorem 1.1] we can use the Baire Category Theorem to show that uniform quasiconformal homogeneity of $E$ implies that there exists a constant $K_{1}$ so that for any two points $s, t \in E$, there exists a $K_{1}$ quasiconformal mapping $f$ of $\hat{\mathbb{C}}$ that keeps $E$ invariant, maps $s$ to $t$, and that fixes $\infty$. Thus, in the proof of [12, Theorem 3.7], the mapping $f$ can in addition be assumed to fix $\infty$, and their proof then yields the stronger conclusion that $E$ is porous. We have thus established:

Lemma 4.1. Let $E \subset \mathbb{R}$ be a uniformly quasiconformally homogeneous compact set. Then $E$ is porous in $\mathbb{R}$.

We are now prepared to prove Theorem 1.2 ,

Proof of Theorem 1.2. Since each of the four statements in the theorem is kept invariant under quasiconformal mappings, we may assume that $E$ is contained in $\mathbb{R}$. By Corollary 2.1, condition (3) is equivalent to the pair $(D, E)$ having the property that $D$ is uniform and $E$ is uniformly perfect. Because $E$ is contained in $\mathbb{R}$, this condition on the pair $(D, E)$ is equivalent by 11 to $E$ being uniformly perfect and $E$ being porous in $\mathbb{R}$. Thus, from now on we will work with the assumption that $E \subset \mathbb{R}$ and, whenever convenient, replace (3) with the following:

$\left(3^{\prime}\right) E$ is uniformly perfect and $E$ is porous in $\mathbb{R}$.

With this normalization we now establish the equivalence of the four statements.

(1) implies (4): This is proved in general for uniformly quasiconformally homogeneous hyperbolic manifolds in [2].

(4) implies $\left(3^{\prime}\right)$ : The lower bound on the injectivity radius function is equivalent to uniform perfectness of $E$ by Pommerenke; see [18, 17.

Next, suppose that $E$ is not porous in $\mathbb{R}$. Then we can find points $x_{n} \in E$ and radii $r_{n}>0$ such that $\left(x_{n}-r_{n}, x_{n}+r_{n}\right) \cap D$ does not contain an interval of length $\frac{r_{n}}{n}$. Define a set $E_{n}=\left(E-x_{n}\right) \cdot \frac{\sqrt{n}}{r_{n}}$ (i.e. shift all points of $E$ by the amount $x_{n}$ and then scale by $\left.\sqrt{n} / r_{n}\right)$, and let $D_{n}=\hat{\mathbb{C}} \backslash E_{n}$. Then $D_{n}$ is conformally equivalent to $D$ for each $n$, and so the injectivity radius function on $D_{n}$ has the same upper and lower bound as the one on $D$. Furthermore, by construction, $0 \in E_{n}$, and $(-\sqrt{n}, \sqrt{n}) \cap D_{n}$ does not contain an interval of length $\frac{1}{\sqrt{n}}$. We claim that as $n \rightarrow \infty$, the injectivity radius in $D_{n}$ at the point $i$ tends to infinity, and this is a contradiction to the existence of a uniform upper bound on the injectivity radius functions on all $D_{n}$. Thus, once this claim is proved, we will know that $E$ must be porous in $\mathbb{R}$. To see that indeed the injectivity radius at $i$ grows larger and larger as 
$n \rightarrow \infty$, observe that for points $z \in D_{n}$ with $\operatorname{Im}(z) \geq \frac{1}{\sqrt{n}}$ and $-\sqrt{n} \leq \operatorname{Re}(z) \leq \sqrt{n}$ we have that $\operatorname{Im}(z) \leq \operatorname{dist}\left(z, E_{n}\right) \leq \sqrt{2} \operatorname{Im}(z)$. Thus for such $z$, the density of the quasihyperbolic metric is uniformly comparable to the density of the hyperbolic metric on $\mathbb{H}^{2}$. As $n \rightarrow \infty$, larger and larger hyperbolic balls (in the hyperbolic metric on $\left.\mathbb{H}^{2}\right)$, centered at $i$, fit into $\left\{z \mid \operatorname{Im}(z) \geq \frac{1}{\sqrt{n}}\right.$ and $\left.-\sqrt{n} \leq \operatorname{Re}(z) \leq \sqrt{n}\right\}$, and so larger and larger quasihyperbolic balls fit into $D_{n}$ as $n \rightarrow \infty$. Hence, arbitrarily large quasihyperbolic balls embed into $D$. But since $E=\hat{\mathbb{C}} \backslash D$ is uniformly perfect, the quasihyperbolic and hyperbolic metrics on $D$ are uniformly comparable, which implies that the hyperbolic injectivity radius function is not bounded above on $D$; a contradiction.

(3) implies (2): Since $\left(3^{\prime}\right)$ and (3) are equivalent, these two conditions clearly imply (2).

(3) implies (1): Again, since $\left(3^{\prime}\right)$ and (3) are equivalent, these two conditions imply (1).

(2) implies $\left(3^{\prime}\right)$ : All that we have to show is that uniform quasiconformal homogeneity of $E \subset \mathbb{R}$ implies porosity of $E$ in $\mathbb{R}$. But this is proved in Lemma 4.1.

We now provide an example that shows that condition (4) in the above theorem, though still necessary for uniform quasiconformal homogeneity of $D$, is no longer sufficient if the assumption of $E$ being contained in a quasicircle is dropped.

Example 4.2. There exists a planar domain $D$ which has bounded geometry but is not uniformly quasiconformally homogeneous.

By $D$ having bounded geometry we mean that there exist two positive constants which bound the injectivity radius function on $D$.

Construction. Let $D_{1}=\hat{\mathbb{C}} \backslash E_{1}$, where $E_{1}$ is a uniformly perfect and porous Cantor set contained in the interval $[-2,-1]$. Let $D_{2}=\hat{\mathbb{C}} \backslash E_{2}$, where $E_{2}$ is contained in the ball of radius 1 , centered at 1 and $E_{2}$ is the limit set of the Klein combination $\Gamma$ of a singly degenerate, purely loxodromic group with a loxodromic cyclic group. Then $D_{2}$ is uniformly quasiconformally homogeneous (it is the regular set of $\Gamma$ and has a compact fundamental domain for the action of $\Gamma$ on $D$ ), and $E_{2}$ is a uniformly perfect compact set of Hausdorff dimension two. Furthermore, each boundary point of $D_{2}$ is the accumulation point of continua in $E_{2}$. Let $D=D_{1} \cap D_{2}=\hat{\mathbb{C}} \backslash\left(E_{1} \cup E_{2}\right)$.

We claim: $D$ has bounded geometry.

To see this, we will first show that $E=E_{1} \cup E_{2}$ is uniformly perfect; this will prove that the injectivity radius function is bounded away from zero on $D$. To prove uniform perfectness, let $A$ be an annulus in $D$, separating $E$. Then $A$ is contained in both $D_{1}$ and $D_{2}$, and if $A$ happens to separate either $E_{1}$ or $E_{2}$, we get the desired bound on the modulus of $A$ by uniform perfectness of $E_{1}$ or $E_{2}$, respectively. Now suppose that $A$ separates neither $E_{1}$ nor $E_{2}$. Then, since $A$ does separate $E$ itself, $E_{1}$ is contained in one component of the complement of $A$, and $E_{2}$ is contained in the other component. Suppose that $E_{1}$ is contained in the bounded component of $A$. Then the diameter of the interior component of $A$ is at least the diameter of $E_{1}$, i.e. it is at least 1 . Furthermore, the distance between inside and outside components of $A$ is at most $\operatorname{dist}\left(E_{1}, E_{2}\right)$ which is at most 3 . Since the modulus of $A$ is bounded by a function of distance over diameter, we have that the modulus of $A$ is bounded above independently of the choice of $A$. This proves that $E$ is uniformly perfect, and thus the injectivity radius function on $D$ is bounded away from zero. 
To prove the existence of an upper bound on the injectivity radius in $D$, observe that by the uniform perfectness of $E$, the quasihyperbolic and hyperbolic metrics in $D$ are uniformly comparable. It thus suffices to show the existence of an upper bound on the quasihyperbolic injectivity radius function on $D$. First observe that for $z \in D$ with $\operatorname{dist}\left(z, E_{1}\right) \leq \frac{1}{2}$ or $\operatorname{dist}\left(z, E_{2}\right) \leq \frac{1}{2}$ we have that $\operatorname{dist}(z, E)=$ $\operatorname{dist}\left(z, E_{1}\right)$ and $\operatorname{dist}(z, E)=\operatorname{dist}\left(z, E_{2}\right)$, respectively. Hence the quasihyperbolic density agrees here with the one in $D_{1}$ and $D_{2}$, respectively, so that the injectivity radius function is bounded from above close enough to $E_{1}$ and $E_{2}$. To see this, recall that the quasihyperbolic injectivity radius function is bounded from above in $D_{1}$ and in $D_{2}$, so through every point in $D_{1}$ and $D_{2}$ there exists a homotopically non-trivial curve of quasihyperbolic length of at most some constant $C$. For points in $D$ that are close enough to $E_{1} \cup E_{2}$, such curves don't leave the Euclidean neighborhood of radius $1 / 2$ of $E_{1} \cup E_{2}$, thus the quasihyperbolic injectivity radius function is bounded from above close enough to $E_{1} \cup E_{2}$.

Suppose now that there are $z_{n} \in D$ with $\operatorname{inj}\left(z_{n}, D\right) \rightarrow \infty$. Passing to a subsequence, we may assume that $z_{n} \rightarrow z_{0} \in \hat{\mathbb{C}}$. Since near $E_{1} \cup E_{2}$ the injectivity radius function is bounded, we must have that $z_{0} \in D$. And thus $\operatorname{inj}\left(z_{0}, D\right)=\infty$. But this cannot be true, as the shortest curve through $z_{0}$, around $E_{1}$, with $E_{2}$ on the outside, has a certain finite hyperbolic length, and thus $\operatorname{inj}\left(z_{0}, D\right)$ is finite. Hence the injectivity radius function is bounded from above on $D$.

We further claim: $D$ is not uniformly quasiconformally homogeneous.

Observe first that if $f$ is a quasiconformal mapping of $\hat{\mathbb{C}}$ preserving $D$, then $f\left(E_{1}\right)$ must be a Cantor set (i.e. a totally disconnected perfect set) contained in $E_{1} \cup E_{2}$, since $E_{1}$ is a Cantor set and since $f(E)=E$. Now let $\alpha$ be the hyperbolic geodesic Jordan curve separating $E_{1}$ and $E_{2}$. Then $f(\alpha)$ is a Jordan curve, one of whose complementary components contains the Cantor set $f\left(E_{1}\right)$ and no other points of $E$. In particular, every point of $f\left(E_{1}\right)$ has a neighborhood that does not contain any continua from $E$. Since $E_{2}$ has the property that continua accumulate at every one of its points, we conclude that $f\left(E_{1}\right)$ must be contained in $E_{1}$.

Suppose now that $D$ is uniformly quasiconformally homogeneous. Fix $z_{0} \in D$ and choose $z_{n} \in D$ such that the $z_{n}$ converge to some point $\zeta$ in $E_{2}$. Since we assumed that $D$ is uniformly quasiconformally homogeneous, there must exist quasiconformal mappings $f_{n}: \hat{\mathbb{C}} \rightarrow \hat{\mathbb{C}}$ preserving $D$ with uniformly bounded dilatation, such that $f_{n}\left(z_{0}\right)=z_{n}$. The restrictions of these $f_{n}$ to $D \cup E_{1}$ form a normal family (they all omit $E_{2}$ ), and since on $D$ no subsequence converges to a homeomorphism of $D$, there must be a subsequence $\left\{f_{n_{j}}\right\}$ converging to $\zeta$ locally uniformly in $D \cup E_{1}=D_{2}$. In particular, since $E_{1}$ is a compact subset of $D_{2}$, we conclude that $f_{n_{j}} \rightarrow \zeta \in E_{2}$ uniformly on $E_{1}$. But this is impossible since $f_{n}\left(E_{1}\right) \subset E_{1}$ as we observed above. Hence, the assumption of homogeneity of $D$ was wrong, and so $D$ is not uniformly quasiconformally homogeneous.

We finish this paper with a question. For a pair $(D, E)$ that is quasiconformally bi-homogeneous (here, $D$ is a domain in $\hat{\mathbb{C}}$ and $E=\hat{\mathbb{C}} \backslash D$ is its complement) we let

$$
\begin{aligned}
K_{D, E} & =\inf \{K \geq 1 \mid(D, E) \text { is } K \text {-quasiconformally bi-homogeneous }\} \\
K_{D} & =\inf \{K \geq 1 \mid D \text { is } K \text {-quasiconformally homogeneous }\} \\
K_{E} & =\inf \{K \geq 1 \mid E \text { is } K \text {-quasiconformally homogeneous }\}
\end{aligned}
$$


Clearly, $K_{D, E} \geq \max \left\{K_{D}, K_{E}\right\}$, and there are examples of pairs $(D, E)$ for which $K_{D, E}>K_{E}$. Such pairs can be constructed for example by observing that there are pairs $(D, E)$ for which $K_{E}$ is arbitrarily close to 1 (see [12]) but $K_{D, E}$ is always bounded away from one (see [13]). We also note that there exists a constant $K_{0}>1$ such that $K_{D} \geq K_{0}$ for all uniformly quasiconformally homogeneous domains $D$ (see [3]). We thus ask:

Question 4.3. Let $(D, E)$ be a quasiconformally bi-homogeneous pair. Is there an upper bound on $K_{D, E}$ in terms of $K_{E}$ and $K_{D}$ ?

\section{REFERENCES}

1. P. Bonfert-Taylor, M. Bridgeman, R.D. Canary and E. Taylor, Quasiconformal homogeneity of hyperbolic surfaces with fixed-point full automorphisms, Math. Proc. Cambridge Philos. Soc. 143 (1) (2007), 71-84. MR2340976 (2008f:30090)

2. P. Bonfert-Taylor, R.D. Canary, G. Martin and E.C. Taylor, Quasiconformal homogeneity of hyperbolic manifolds, Math. Ann. 331 (2005), 281-295. MR2115457(2006b:30042)

3. P. Bonfert-Taylor, R.D. Canary, G. Martin, E.C. Taylor and M. Wolf, Ambient quasiconformal homogeneity of planar domains, preprint.

4. P. Bonfert-Taylor, G. Martin, A. Reid and E. Taylor, Teichmüller mappings, quasiconformal homogeneity, and non-amenable covers of Riemann surfaces, to appear in Pure and Applied Mathematics Quarterly.

5. P. Bonfert-Taylor and E. Taylor, Quasiconformal homogeneity of limit sets, preprint.

6. F.W. Gehring and B. Palka, Quasiconformally homogeneous domains, J. Analyse Math., 30 (1976), 172-199. MR0437753 (55:10676)

7. P. Jones, and S. Smirnov, Removability theorems for Sobolev functions and quasiconformal maps, Ark. Mat. 38 (2000), 263-279. MR.1785402 (2001i:46049)

8. R. Kaufman, and J.-M. Wu, On removable sets for quasiconformal mappings, Ark. Mat. 34 (1996), 141-158. MR.1396628 (97f:30029)

9. P. Koskela, Removable sets for Sobolev spaces, Ark. Mat. 37 (1999), 291-304. MR 1714767 (2001g:46077)

10. P. Koskela, and T. Nieminen, Quasiconformal Removability and The Quasihyperbolic Metric, Indiana Univ. Math. J. 54 (2005), no. 1, 143-151. MR.2126078 (2005k:46079)

11. P. MacManus, Catching sets with quasicircles, Rev. Mat. Iberoamericana 15 (1999), no. 2, 267-277. MR1715408(2000h:30031)

12. P. MacManus, R. Näkki and B. Palka, Quasiconformally Homogeneous Compacta in the Complex Plane, Michigan Math. J. 45 (1998), 227-241. MR 1637642(99k:30031)

13. P. MacManus, R. Näkki and B. Palka, Quasiconformally bi-homogeneous compacta in the complex plane, Proc. London Math. Soc. (3) 78 (1999), no. 1, 215-240. MR1658172 $(99 \mathrm{j}: 30020)$

14. B. Maskit, Kleinian groups. Grundlehren der Mathematischen Wissenschaften 287. SpringerVerlag, Berlin, 1988. MR959135 (90a:30132)

15. C. McMullen, Kleinian groups and John domains, Topology 37 (1998), no. 3, 485-496. MR.1604874 (99c:20070)

16. R. Näkki and J. Väisälä, John disks, Exposition. Math. 9 (1991), no. 1, 3-43. MR1101948 (92i:30021)

17. Ch. Pommerenke, On uniformly perfect sets and Fuchsian groups, Analysis 4 (1984), no. 3-4, 299-321. MR.780609 (86e:30044)

18. Ch. Pommerenke, Uniformly perfect sets and the Poincaré metric, Arch. Math. (Basel) 32 (1979), no. 2, 192-199. MR.534933 (80j:30073)

Department of Mathematics, Wesleyan University, Middletown, Connecticut 06459

E-mail address: pbonfert@wesleyan.edu

Department of Mathematics, Wesleyan University, Middletown, Connecticut 06459

E-mail address: ectaylor@wesleyan.edu 